\title{
The Distribution of Bar Strengths in Disk Galaxies
}

\author{
R. Buta ${ }^{1}$, E. Laurikainen ${ }^{2}$, H. Salo ${ }^{2}$, J. H. Knapen ${ }^{3}$, and D. L. Block ${ }^{4}$ \\ ${ }^{1}$ Dept. of Physics \& Astronomy, University of Alabama, Tuscaloosa, AL 35487, USA \\ ${ }^{2}$ Division of Astronomy, Dept. of Physical Sciences, University of Oulu, FIN-90014, Finland \\ ${ }^{3}$ University of Hertfordshire, Centre for Astrophysics Research, Hatfield, Herts AL10, 9AB, UK \\ ${ }^{4}$ School of Computational \& Applied Mathematics, University of the Witwatersrand, \\ Johannesburg, South Africa
}

\begin{abstract}
Bars are the most important features that redistribute angular momentum and drive secular evolution in disk galaxies. We have derived the distribution of bar strengths in spirals and the Fourier properties of early-type galaxy bars in order to evaluate recent models of bar-halo angular momentum transfer and external gas accretion. A few results are presented here.
\end{abstract}

Keywords. galaxies: bars, galaxies: evolution

The Fourier properties of bars and the distribution of bar strengths in disk galaxies provide important tests of models of the evolution of bars, such as the halo angular momentum transfer idea of Athanassoula (2003, MNRAS, 341, 1179) and the external gas accretion mechanism of Bournaud \& Combes (2002, A\&A, 392, 83). Figure 1 shows some of our recent results connected with these issues. This work has been supported by NSF grants AST020-5143 and AST050-7140, the Academy of Finland, the Leverhulme Trust, and the Anglo-American Chairman's Fund.
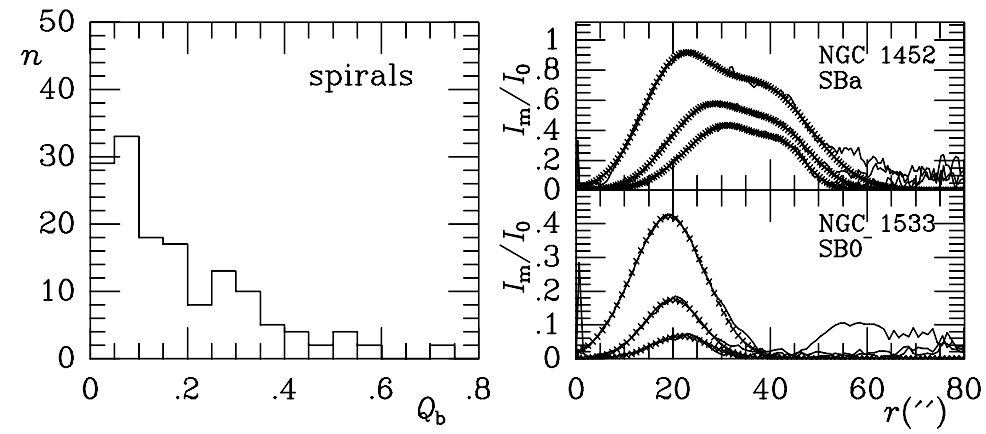

Figure 1. Left: Distribution of maximum relative gravitational bar torques for 147 spiral galaxies (Buta et al. 2005, AJ, 130, 506). This kind of plot may contain information on possible recurrent bar formation in systems accreting gas (Block et al. 2002, A\&A, 394, L35). Right: Relative Fourier intensity profiles of $m=2,4,6$ components (top to bottom curves, respectively) of the bars of the early-type galaxies NGC 1452 and 1533 (Buta et al. 2006, in press, astro/ph-0609406). The profiles for NGC 1452 are well-fitted by a double gaussian (crosses) and strongly resemble Athanassoula's n-body models where the bar transfers a large amount of angular momentum to a massive halo. In contrast, NGC 1533 has profiles best described in terms of a single gaussian, suggesting possibly a disk-dominated system. 